\title{
Drug loading and release behaviors of freestanding polysaccharide composite films
}

\author{
Mineo Hashizume ${ }^{1,2}$, Yu Murata ${ }^{2}$, Kazutoshi Iijima ${ }^{1,2}$ and Tomonori Shibata ${ }^{2}$ \\ We developed freestanding, water-insoluble thin films made of polyion complexes (PICs) that consist of glycosaminoglycans such \\ as chondroitin sulfate $\mathrm{C}$ (CS) and chitosan (CHI) by utilizing hot press techniques. In this study, material loading and release \\ abilities of $\mathrm{CS} / \mathrm{CHI}$ composite films were spectroscopically examined using small dye molecules; cationic methylene blue (MB) \\ and anionic carboxyfluorescein (CF) were used as model drugs. The dye-loading films were prepared using pre- and post-loading \\ methods. Microscopic film morphologies were not affected by the dye loadings. The mechanical strengths of the dye-loaded \\ $\mathrm{CS} / \mathrm{CHI}$ films were almost identical to those of the unloaded films. The dye release behaviors of the films in various aqueous \\ solutions were examined, and the results demonstrated that the $\mathrm{CS} / \mathrm{CHI}$ films exhibited media-responsive dye release abilities. \\ The experimental results obtained under different conditions were compared and discussed based on the chemical structures of \\ the polysaccharides used to generate the film components and the dye molecules. The present results demonstrate that these \\ polysaccharide composite films are promising materials for use in applications that require sustained drug release under \\ physiological conditions.
}

Polymer Journal (2016) 48, 545-550; doi:10.1038/pj.2015.126; published online 13 January 2016

\section{INTRODUCTION}

Stimuli-responsive polymer materials have recently gained much attention because of their potential applications in various fields. ${ }^{1}$ Much effort has been devoted to design and synthesize such novel synthetic polymers with various main chain structures, functional groups, molecular lengths and branching; there have also been efforts to fabricate nanodevices and materials much larger in size using these polymers. In biomedical fields, one promising application of these stimuli-responsive polymers is their use as drug delivery systems (DDS). ${ }^{2}$ In addition to nano-sized and colloidal polymer particles, polymer films responsive to surrounding physiological conditions are useful. They can be used as fomentations, plasters, wound dressings, and coatings for medical devices that have controlled drug release abilities. $^{3,4}$ For such purposes, the preparation of stimuli-responsive polymer film materials made of natural polymers is attractive because of their biocompatibility and biodegradability; the latter can be utilized as a trigger for drug release. The utilization of biopolymers as structural materials, such as films, is also interesting from the viewpoint of the utilization of biomass. Natural polysaccharides are a candidate biopolymer because some of their industrial supplies have been established. The utilization of natural polysaccharides as biomaterials has been investigated ${ }^{5-8}$ and they have also been used to prepare films and bulk solids or gels with drug-release abilities. ${ }^{9-14}$ However, in most cases, they were chemically modified to reduce water solubility. Such treatments could reduce the intrinsic biocompatibility of the polysaccharides.
Based on these previous studies, we have been developing structural materials from natural polysaccharides with a special focus on the development of films. As a result, we developed freestanding polysaccharide composite films made of polyion complexes (PICs) that consist of glycosaminoglycans such as chondroitin sulfate (CS) and chitosan (CHI) by utilizing hot press techniques. ${ }^{15,16}$ These films have been shown to possess sufficient mechanical strength and swelling behavior in water but are basically water-insoluble even though they are only made of water-soluble polysaccharides. Such properties are important for their use as materials in sustained drug release applications. The polysaccharide-based PICs can also be formed into fibers. ${ }^{17}$

In this study, the use of polysaccharide composite films as sustained drug release materials was evaluated. First, small cationic and anionic dye molecules were used as model drugs to prepare dye-loaded polysaccharide composite films. The dye release properties of the resulting films were then examined. The effects of sample preparation and release conditions on loading amounts and release behaviors of the films were also investigated.

\section{MATERIALS AND METHODS}

\section{Materials}

Methylene blue (MB) was obtained from Kanto Chemical Co., Inc. 5 (6)-Carboxyfluorescein (CF) and 4-methylumbelliferyl- $\beta$-D-galactopyranoside (MUG) were purchased from Wako Pure Chemical Industries, Ltd. CS (sodium salt, from shark cartilage, MW ca. 20 000), CHI (from crab shell,

\footnotetext{
${ }^{1}$ Department of Industrial Chemistry, Faculty of Engineering, Tokyo University of Science, Tokyo, Japan and ${ }^{2}$ Graduate School of Chemical Sciences and Technology, Tokyo University of Science, Tokyo, Japan

Correspondence: Professor M Hashizume, Department of Industrial Chemistry, Faculty of Engineering, Tokyo University of Science, 12-1 Ichigayafunagawara-machi, Shinjuku-ku, Tokyo 162-0826, Japan.

E-mail: mhashizu@ci.kagu.tus.ac.jp

Received 30 October 2015; revised 16 November 2015; accepted 18 November 2015; published online 13 January 2016
} 


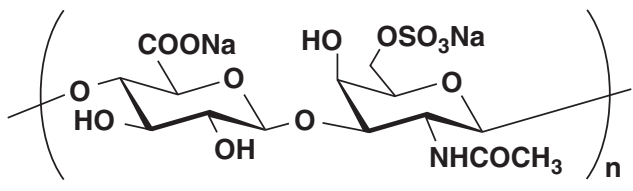

CS

(Sodium salt)

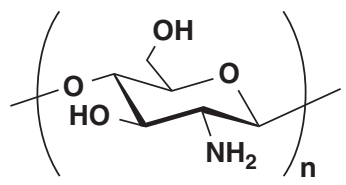

$\mathrm{CHI}$

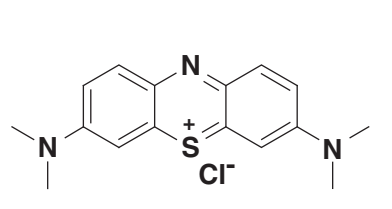

MB<smiles>O=C(O)c1ccc2c(c1)C1(OC2=O)c2ccc(O)cc2OC2C=C(O)C=CC21</smiles>

CF<smiles>Cc1cc(=O)oc2cc(OC(O)C(O)OCC(O)C(O)CO)ccc12</smiles>

MUG

(6-Isomer)

Figure 1 Chemical structures and abbreviations of the polysaccharides and dye molecules used for this study.

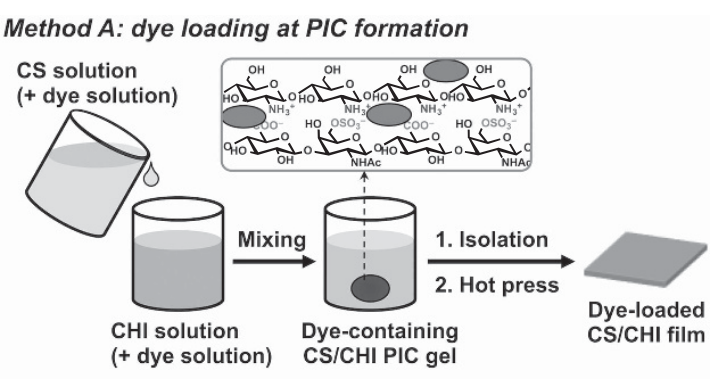

Method B: dye loading after film formation

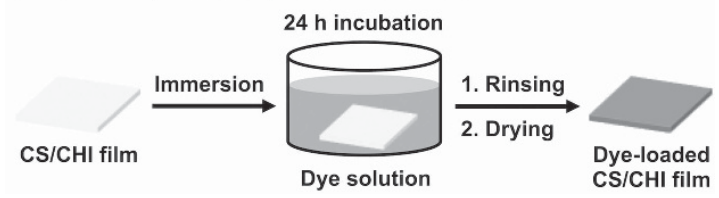

Figure 2 Schematic illustration of the dye-loaded $\mathrm{CS} / \mathrm{CHI}$ film preparation processes. A full color version of this figure is available at Polymer Journal online.

$M W \geqslant 100000$ ), and other chemicals were obtained from Nacalai Tesque Inc. The chemical structures and abbreviations of these molecules are summarized in Figure 1. All chemicals were used as received. Distilled water was prepared for experiments (RFD210TA; Advantec Toyo Kaisha Ltd.).

\section{Preparation of dye-loaded polysaccharide composite films}

All of the experiments in this study were conducted at room temperature (approximately $25^{\circ} \mathrm{C}$ ) unless otherwise stated. Polysaccharide composite films made of PICs of CS and CHI (hereafter denoted as CS/CHI) that did not load dye molecules were prepared using the same procedure that was reported in one of our previous publications ('method B' in the paper). ${ }^{16}$ Dye-loaded $\mathrm{CS} / \mathrm{CHI}$ films were prepared using two methods (Figure 2). In Method A, where dye molecules were loaded at the time of PIC formation, aqueous solutions of CS (2.0 wt.\% as sodium salts) were added dropwise to an aqueous acetic acid (1.0 wt.\%) solution of CHI (1.0 wt.\%) until the formation of gel-like CS/CHI PICs was completed. Here, solutions of the dye molecules were added to the CS or CHI solutions before mixing. Representative conditions, sample names and corresponding solution conditions are listed in Table 1. The obtained PICs were collected using centrifugation (6000 r.p.m., 3 min) and by washing the samples with distilled water; a longer centrifugation period was used (6000 r.p.m., $10 \mathrm{~min}$ ) for their isolation. The PICs could also be isolated using lyophilization instead of a longer centrifugation period ('method A' in the reference paper). ${ }^{16}$ The resulting dye-loaded PICs were then hot pressed to form freestanding films using the same procedure to prepare the $\mathrm{CS} / \mathrm{CHI}$ films $\left(120^{\circ} \mathrm{C}, 20 \mathrm{MPa}, 3 \mathrm{~min}\right)$. Film thicknesses were controlled to be $100 \mu \mathrm{m}$ using a poly(ethylene terephthalate) (PET) spacer with rectangular holes ( $1 \mathrm{~cm} \times 1 \mathrm{~cm}-5 \mathrm{~cm} \times 5 \mathrm{~cm}$ depending on the amount of gel). The resulting films were cut into desired sizes (for example, $1 \mathrm{~cm} \times 1 \mathrm{~cm}$ ) using a cutter knife.

In Method B, the CS/CHI films $(1 \mathrm{~cm} \times 1 \mathrm{~cm})$ were immersed in each dye solution $\left(1.0 \times 10^{-3} \mathrm{M}, 50 \mathrm{ml}\right)$ for $24 \mathrm{~h}$ at room temperature (Figure 2, Table 1). After removing the films from the solution, the films were rinsed with distilled water. The remaining water was removed with a laboratory wipe, and the films were dried in air for $24 \mathrm{~h}$ and used for the experiments.

\section{Characterization}

Standard characterization of the films, such as morphological observation using scanning electron microscopy (SEM) (JSM-7001F; JEOL Ltd.), FT-IR measurements (Nicolet 380; Thermo Fisher Scientific Inc., equipped with a single reflection ATR module (Smart Orbit)), and tensile strength measurements (Autograph AGS-500NJ; Shimadzu Corp.), were conducted using the same methods that were reported previously. ${ }^{16}$

The swelling behaviors of the films in water and buffers were also conducted using previously reported procedures. ${ }^{16,18}$ The degree of swelling (\%) and weight loss (\%) were calculated using equations 1 and 2, respectively.

Degree of swelling $(\%)=\left(W_{\mathrm{S}}-W_{\mathrm{I}}\right) / W_{\mathrm{I}} \times 100$

Weight $\operatorname{loss}(\%)=\left(W_{\mathrm{I}}-W_{\mathrm{F}}\right) / W_{\mathrm{I}} \times 100$

where $W_{\mathrm{I}}$ stands for the initial weight of the film $(1 \mathrm{~cm} \times 1 \mathrm{~cm})$ before immersion, $W_{S}$ represents the weight of the swelled film after immersion ( $30 \mathrm{~min}$ ), and $W_{\mathrm{F}}$ signifies the final weight of the film after the immersion experiments followed by drying in air.

Evaluation of dye loading and release abilities of the films

The dye-loading amounts were evaluated using a UV-vis spectrophotometer (V-660; JASCO). Dye solutions before using the treatments, the supernatant of the mixtures after PIC formation (Method A) and the dye solutions after film immersions (Method B) were examined. Aliquots of the sample solutions were diluted by 100 times and the spectra were recorded. The absorption maxima of MB, CF, and MUG used for the calculation of dye amounts were 665, 492, and $315 \mathrm{~nm}$, respectively. The differences in the absorbances between these solutions were used for the calculations. More than two pieces of films were examined for each sample.

The release behaviors of the dye molecules from the films were also examined using UV-vis spectroscopy. Dye-loaded films $(1 \mathrm{~cm} \times 1 \mathrm{~cm})$ were immersed in different types of solutions: distilled water, phosphate buffered saline (PBS, pH 7.4), and phosphate buffer (PB) prepared using $\mathrm{Na}_{2} \mathrm{HPO}_{4}$ and $\mathrm{NaH}_{2} \mathrm{PO}_{4}$ (corresponded to $100 \mathrm{~mm}$ of phosphate ion species, $\mathrm{pH} 5.8,7.4$, and 8.0). The samples were then incubated at $36.5^{\circ} \mathrm{C}$ using a dry chamber 
Table 1 Solution conditions and sample names for preparation of dye-loaded CS/CHI films

Method A: solutions added to

\begin{tabular}{|c|c|c|c|}
\hline $\mathrm{CS} / \mathrm{CHI}$ & - & - & - \\
\hline MB-A & - & + MB solution ${ }^{c}$ & - \\
\hline MB5-A & - & + MB solution ${ }^{d}$ & - \\
\hline$M B^{\prime}-A$ & + MB solution ${ }^{\mathrm{c}, \mathrm{e}}$ & - & - \\
\hline CF-A & + CF solution $^{f}$ & - & - \\
\hline CF'-A & - & $+\mathrm{CF}$ solution ${ }^{f}$ & - \\
\hline MUG-A & - & + MUG solutiong & - \\
\hline MUG'-A & + MUG solutiong & - & - \\
\hline MB-B & - & - & MB solution $\left(1.0 \times 10^{-3} \mathrm{M}, \mathrm{pH} 4.8\right)$ \\
\hline CF-B & - & - & CF solution $\left(1.0 \times 10^{-3} \mathrm{M}, \mathrm{pH} 11.4\right)$ \\
\hline MUG-B & - & - & MUG solution $\left(1.0 \times 10^{-3} \mathrm{M}, \mathrm{pH} 5.1\right)$ \\
\hline
\end{tabular}

a2 wt.\%, $7 \mathrm{ml}$.

b1 wt.\% in aqueous acetic acid (1 wt.\%), $10 \mathrm{ml}$

c $1.0 \times 10^{-3} \mathrm{M}, \mathrm{pH} 4.8,10 \mathrm{ml}$.

d $1.0 \times 10^{-3} \mathrm{M}, \mathrm{pH} 4.8,5 \mathrm{ml}$.

${ }^{\mathrm{e}} \mathrm{CS}$ solutions was $9 \mathrm{ml}$.

${ }^{\mathrm{f}} 1.0 \times 10^{-3} \mathrm{M}, \mathrm{pH} 11.4,10 \mathrm{ml}$

$\mathrm{g} 1.0 \times 10^{-3} \mathrm{~m}, \mathrm{pH} 5.1,10 \mathrm{ml}$.
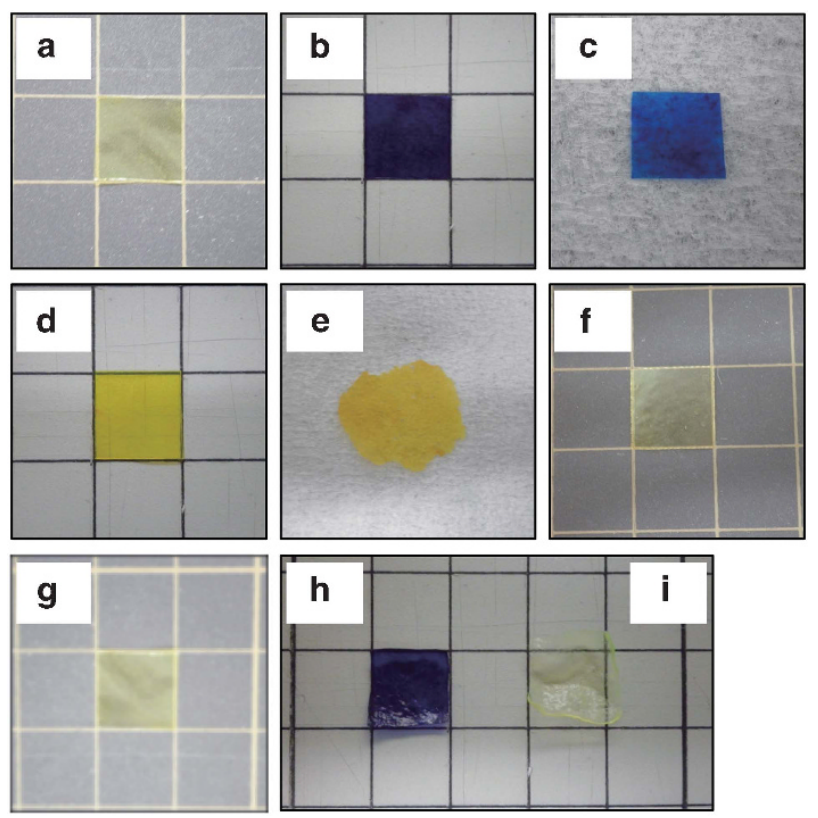

Figure 3 Macroscopic images of dye-loaded $\mathrm{CS} / \mathrm{CHI}$ films. (a) $\mathrm{CS} / \mathrm{CHI}$, (b) MB-A, (c) MB'-A, (d) CF-A, (e) CF'-A, (f) MUG-A, (g) MUG'-A, (h) MB-B, and (i) CF-B films. In some images, the films were placed on a cutting mat with $1 \mathrm{~cm}$-line grids.

(DOV-750A; AS ONE) under gentle stirring. Teflon ${ }^{\infty}$ vials were used to prevent dye adsorption to glass vials at high concentrations. The UV-vis spectra of the solutions were recorded using a similar method as the procedure described above at suitable intervals.

\section{RESULTS AND DISCUSSION}

\section{Preparation of dye-loaded CS/CHI films}

In Method $\mathrm{A}$, dye molecules were added to a polysaccharide solution prior to mixing with the counterpart solution. Both the addition of dye molecules to the solution of the polysaccharide with the same charges as the dyes and that those with opposite charges were examined (Table 1). This was because ion complex formation between the dyes and the polysaccharides with opposite charges could affect the formation of the PIC and/or the resulting films.

MB-A, where $\mathrm{MB}$ solutions were added to $\mathrm{CHI}$ solutions before mixing with CS solutions, showed similar gel isolation yields ( $c a .85 \%)$ to that of CS/CHI PICs without the addition of dyes. ${ }^{16}$ Macroscopic images of the films also confirmed that the MB-A films had similar macroscopic morphologies to those of the CS/CHI films, except for the fact that they were colored as a result of the incorporation of $\mathrm{MB}$ molecules (Figures 3a and b). MB5-A, which contained a smaller amount of $\mathrm{MB}$, showed less-colored films, as expected (data not shown). For MB'-A, where MB solutions were added to CS solutions before addition to $\mathrm{CHI}$ solutions, a higher amount of the CS solutions were needed to complete PIC formation compared to the procedure used to generate MB-A (Table 1). This result supported that idea that the anionic charges of CS used for PIC formation with CHI were partially consumed by the interaction with MB. Despite this, similar films were finally obtained, as shown in Figure 3c.

As for $\mathrm{CF}$, an anionic dye, premixing of CF solutions with the CS and $\mathrm{CHI}$ solutions are denoted as $\mathrm{CF}-\mathrm{A}$ and $\mathrm{CF}$-A, respectively (Table 1). The yields of the corresponding PIC were $c a .80 \%$ for CF-A and approximately $40 \%$ for CF'-A. CF-loaded films were successfully obtained for CF-A (Figure 3d). On the other hand, the PICs obtained from CF'-A were hardly formed into thin films (Figure $3 \mathrm{e}$ ). The results for CF'-A supported the fact that electrostatic interactions between $\mathrm{CF}$ and $\mathrm{CHI}$ in the premixing solutions largely affected PIC and film formation. The reason $\mathrm{CF}$ exhibited a larger effect than $\mathrm{MB}$ could be due to the effects resulting from differences such as molecular size, potential multivalency (CF can form dianionic structures under basic conditions), and solution $\mathrm{pH}$ (for $\mathrm{CF}$ systems, solutions became more basic than MB systems after mixing).

MUG was used as a reference nonionic molecule, and MUG-loaded $\mathrm{CS} / \mathrm{CHI}$ films were prepared using the same experimental procedure. For expediential reasons, MUGs premising $\mathrm{CHI}$ and $\mathrm{CS}$ were denoted as MUG-A and MUG'-A, respectively (Figures $3 \mathrm{f}$ and $\mathrm{g}$ ). For both cases, the corresponding resulting freestanding films were successfully obtained, but incorporation of MUG in the films was difficult to confirm because of its relatively low absorbance in the visible light regions and the low incorporation amounts (will be described later). 
Subsequently, Method B, where the dye was loaded after film formation, was examined for MB, CF, and MUG. The appearance of the resulting films strongly supported the successful incorporation of $\mathrm{MB}$ in the CS/CHI films (MB-B, Figure 3h). Preliminary optical microscopic observations of the cross-section of the films also showed that the MB molecules were distributed not only in the surface regions but also inside of the films; these observations suggested that more MB molecules existed in the former. On the other hand, $\mathrm{MB}$ molecules were distributed homogeneously across all of the film regions in the case of MB-A. Here, the weight loss of the films after immersion in the MB solutions was $1.1 \pm 1.1 \%$ (mean \pm standard deviation), which was the same degree as observations of films immersed in distilled water $(1.7 \pm 0.81 \%)$ and in MUG solutions $(1.8 \pm 0.92 \%)$. On the other hand, CF-loaded films tended to shrink after immersion and drying in air (Figure 3i). In addition, large weight loss $(46 \pm 1.4 \%)$ was observed. This was probably due to the basicity of the CF solutions. We concluded that Method B was not suitable for the preparation of CF-loaded CS/CHI films.

Figure 4 shows the results of the physical characterization of the MB-A films. They were comparable to those of the CS/CHI films without dye loading. ${ }^{16}$ Microscopic morphologies obtained using SEM confirmed dense film structures and smooth film surfaces (Figures $4 \mathrm{a}$ and b). In the FT-IR spectra (Figure 4c), MB loading did not cause
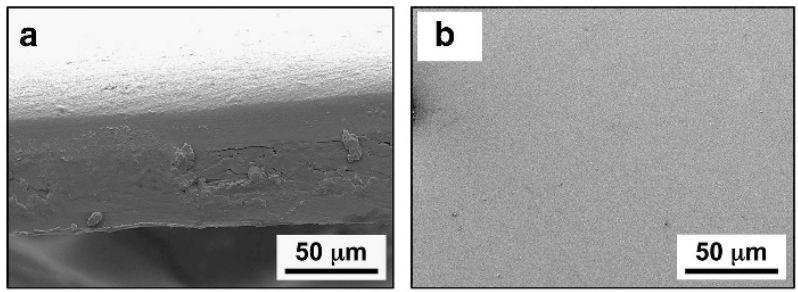

C
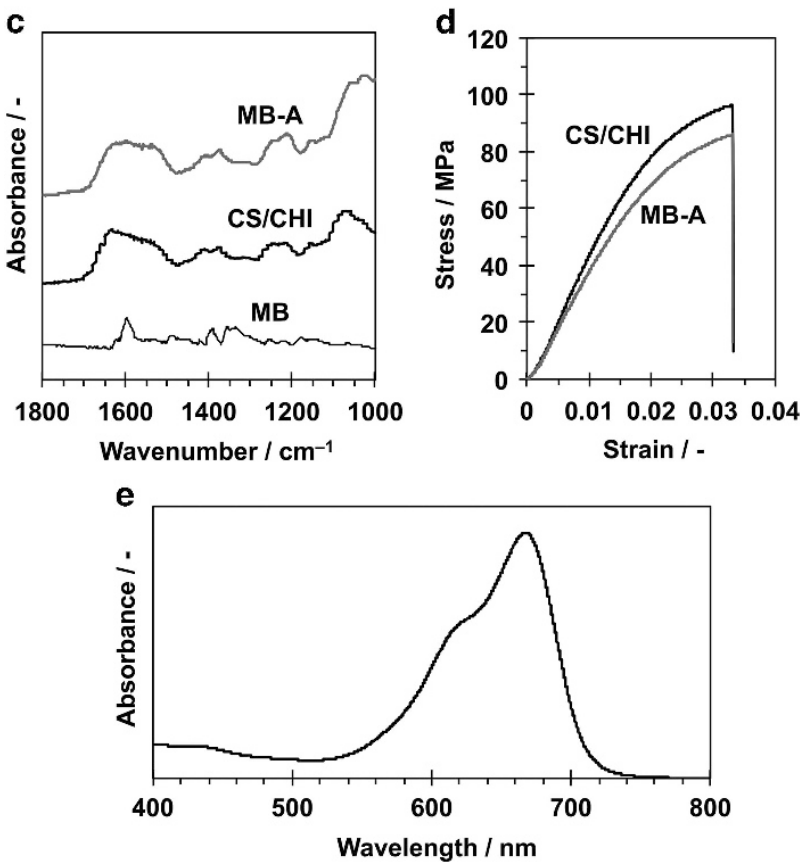

Figure 4 (a, b) SEM images of the cross-section (a) and surface (b) of an MB-A film. (c) FT-IR spectra of the MB, CS/CHI film, and MB-A films. (d) Stress-strain curves of the $\mathrm{CS} / \mathrm{CHI}$ film and MB-A films. (e) UV-vis differential spectra between the MB-A and $\mathrm{CS} / \mathrm{CHI}$ films. For these samples, PIC gels were isolated using lyophilization before hot pressing. A full color version of this figure is available at Polymer Journal online. significant peak changes but we confirmed that the peak at $c a$. $1530 \mathrm{~cm}^{-1}$ that supported PIC formation ${ }^{15,16,19,20}$ was not affected by $\mathrm{MB}$ loading. Tensile strength measurements indicated that $\mathrm{MB}$ loading may have slightly decreased the mechanical strengths of the films but clearly showed that MB-loaded films still possessed sufficient mechanical strength (Figure 4d). Further examinations are needed to judge the significance of the decrease caused by $\mathrm{MB}$ loading. For these characterizations, CF-A films also exhibited similar results (data not shown), which demonstrated that CF incorporation did not affect these film properties. MB incorporation was also confirmed using UV-vis differential spectral measurements (Figure 4e). The resulting spectra resembled that of the MB solutions, ${ }^{21}$ which also demonstrated that the electronic state of $\mathrm{MB}$ was not affected by being incorporation into the CS/CHI films. Unfortunately, high-resolution differential spectra were not always obtained due to technical reasons and variations in the thickness of the film samples.

Dye loading amounts of MB-A, CF-A, and MB-B films were calculated from the spectra of the dye solutions before and after the treatments. Films that were $1 \mathrm{~cm} \times 1 \mathrm{~cm}$ in size were used for the experiments. Evaluation of the MUG-loaded films was not conducted because of the low incorporation of MUG as judged by preliminary experiments. Low incorporation of nonionic molecules indicated that electrostatic interactions contributed to the loading of the molecules into the $\mathrm{CS} / \mathrm{CHI}$ films.

In Method $\mathrm{A}$, the loading amounts of $\mathrm{MB}$ in the MB-A films and those of $\mathrm{CF}$ in the CF-A films were $(3.6 \pm 0.05) \times 10^{-7} \mathrm{~mol}$ and $(3.7 \pm 0.01) \times 10^{-7} \mathrm{~mol}$, respectively. The results supported that the differences in charge and molecular shape between MB and CF did not affect their loading amounts under the present conditions. On the other hand, the loading amount of $\mathrm{MB}$ in the MB-B films was calculated to be $(1.5 \pm 0.01) \times 10^{-6} \mathrm{~mol}$. Method B achieved larger dye loading amounts. Not all of the dyes in the starting solutions were loaded in the films for both methods. The loading amounts seemed to be saturated with respect to the dye concentrations of the treatment solutions.

\section{Dye release behaviors of the CS/CHI films}

Figure $5 \mathrm{a}$ shows the dye release profiles of MB-A, CF-A, and MB-B in distilled water. It is clear that $\mathrm{MB}-\mathrm{B}$ released more $\mathrm{MB}$ molecules and showed a faster release of the molecules than MB-A. In addition, CF-A showed faster dye release behavior than MB-A but their saturated release amounts were similar. The release amounts corresponded to the loading amounts, as described above. It took a few hours for CF-A to reach an equilibrium, whereas it took approximately $24 \mathrm{~h}$ for MB-A and MB-B. The results indicated that the dye release rates were affected by the concentrations and molecular structures of the dyes.

In the case of the dye release behavior in PBS ( $\mathrm{pH} 7.4)$, a remarkable difference was observed in the timescale compared to the dye release profile observed in distilled water (Figure 5b). The dye release profile reached equilibrium in approximately $30 \mathrm{~min}$ for MB-A and CF-A, and approximately $60 \mathrm{~min}$ for MB-B. Such fast release profiles have not been reported for the release of materials from PIC-type substances. For example, more than $60 \mathrm{~h}$ were needed to for the release of silver sulfadiazine from a PIC membrane of CHI and alginate in phosphate buffers to reach equilibrium, ${ }^{22}$ and this release behavior continued for much longer periods in the case of protein release from CS/CHI nanoparticles in PBS. ${ }^{20}$ There was a difference in the degree of swelling between CS/CHI films immersed in distilled water and in PBS (Figure 6). However, this difference was not sufficient to explain the large differences in release rates. For both cases, swelling of the films reached their corresponding equilibria 

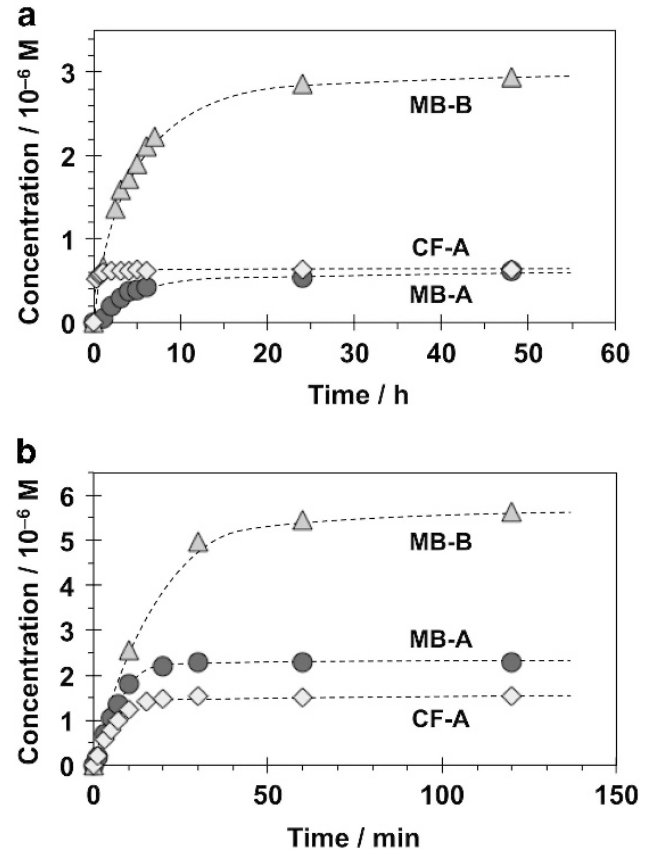

Figure 5 Dye release behaviors of the MB-A (circles), CF-A (rhombuses), and MB-B (triangles) films in distilled water (a) and in PBS ( $\mathrm{pH}$ 7.4) (b). A full color version of this figure is available at Polymer Journal online.

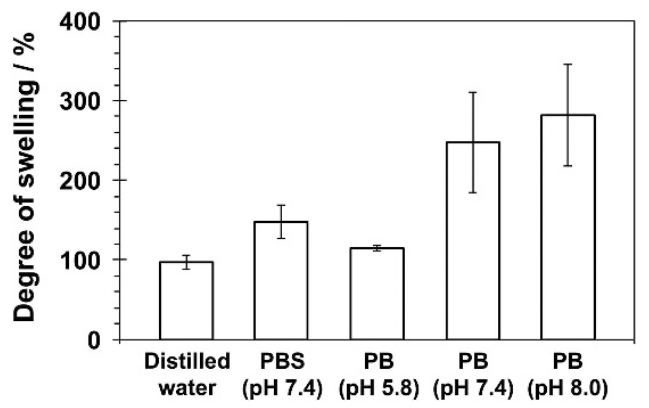

Figure 6 Degrees of swelling of the $\mathrm{CS} / \mathrm{CHI}$ films after 30 min incubation periods in different buffers.

within $30 \mathrm{~min}$. Therefore, it was difficult to explain the slow dye release behavior in distilled water only using the differences in the degrees of swelling. Ionic strength was another factor that may have made such a difference because it affects electrostatic interactions. Differences in swelling degrees and ionic strengths also contributed to the dye release amounts, which appeared larger in PBS than in distilled water. These tendencies were also reported for $\mathrm{CS} / \mathrm{CHI}$ nanoparticles. ${ }^{20}$ According to the results from measurements of the dye loading amounts, the dye release ratios of the MB-A, CF-A, and MB-B films at equilibrium were calculated to be approximately 30 , 20 and $17 \%$, respectively. The reason for these low release ratios remains unclear. One possibility is that parts of the dye molecules interacted strongly with the oppositely charged polysaccharides in the films even under the swollen conditions. Another possibility is that the dye loading amounts obtained by the calculation may appear larger than the actual amounts. The absorbances of the dye solutions after the treatments (PIC formation or film immersion) may have been affected by the polysaccharide molecules released into the solutions during the treatments, although they were much smaller than the amounts of PICs or films in the solutions. In addition, some dye

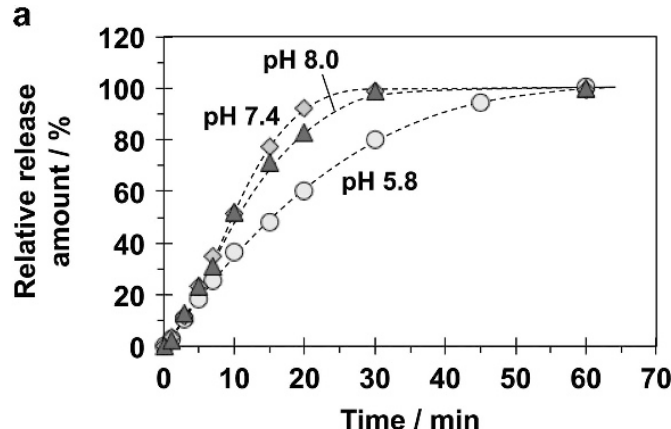

b

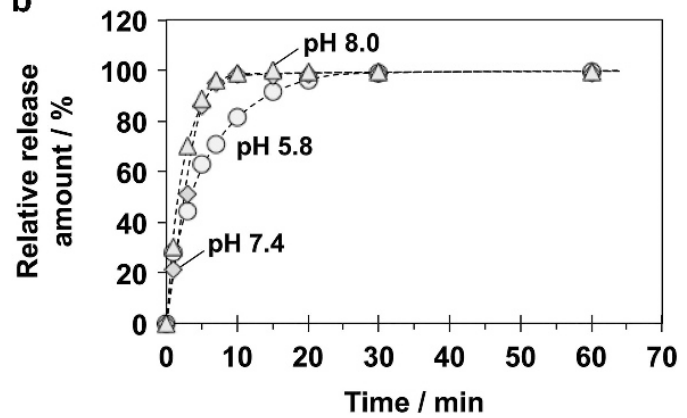

Figure $7 \mathrm{MB}$ (a) and $\mathrm{CF}$ (b) release behaviors from the MB-A (a) and CF-A films (b) in PBS at $\mathrm{pH} 5.8$ (circles), $\mathrm{pH} 7.4$ (rhombuses), and $\mathrm{pH} 8.0$ (triangles). Release amounts were expressed as relative values (cumulative release amounts at equilibrium were normalized to $100 \%$ release). A full color version of this figure is available at Polymer Journal online.

molecules may have been lost during the rinsing process of the PICs or films after picking removing them from the solutions.

There were other differences between the results obtained from films immersed in PBS and in distilled water. The differences in the initial release rates between $\mathrm{MB}-\mathrm{A}$ and $\mathrm{CF}-\mathrm{A}$ were smaller, and the $\mathrm{MB}$ release amounts were larger in PBS. Including these observations, there are several points that must be clearly explained based on the properties of the films and the dyes. Further systematic investigations are currently being conducted in our group.

Finally, the effect of media $\mathrm{pH}$ on the dye release rates was evaluated using $\mathrm{PBs}$ of different $\mathrm{pH}$ values (Figure 7). Here, the results are expressed as relative released amounts. The actual release amounts in PBs were similar to those in PBS (Figure 5) and no significant differences were observed. This is reasonable when considering the ion concentrations of these buffers (PB: $100 \mathrm{~mm}$ of phosphate ions species, PBS: $10 \mathrm{~mm}$ of phosphate ion species $+137 \mathrm{~mm}$ of $\mathrm{NaCl}$ ). Figure 7 clearly demonstrates two points: One is that the CF release rates from the CF-A films (Figure 7b) were faster than the MB release rates from the MB-A films (Figure 7a). Another point is that dye release rates at $\mathrm{pH} 7.4$ and $\mathrm{pH} 8.0$ were faster than those at $\mathrm{pH} 5.8$ for both the MB-A and CF-A films. The latter observation was comparable to that obtained using different degrees of swelling from these solutions (Figure 6). Since the $\mathrm{p} K_{\mathrm{a}}$ of the amino groups of $\mathrm{CHI}$ is $6.5,{ }^{23}$ the $\mathrm{pH}$ change from 5.8 to 8.0 decreased numbers of ammonium ion groups in the $\mathrm{CHI}$ molecules. On the other hand, the degrees of dissociation of the sulfate and carboxylic acid groups were not significantly affected when the $\mathrm{pH}$ was changed from 5.8 to 8.0. Therefore, it was suggested that the number of ion complexes decreased in the films by making the films more anionic by changing the $\mathrm{pH}$ from 5.8 to 8.0 ; these changes were able to affect the swelling degrees of the films and the electrostatic interactions between the dye molecules and the films. The 
$\mathrm{pH}$ dependent increases of swelling degrees and corresponding materials releases were also observed for the crosslinked $\mathrm{CHI} /$ silk fibroin blend films ${ }^{24}$ and hyaluronic acid/polyethyelenimine PIC nanoparticles $^{25}$ (both of these were designed to increase swelling and release rates by at lower $\mathrm{pH}$ values). This discussion is also useful for explaining the results of the first point, i.e., faster CF release compared to $\mathrm{MB}$. With an increase of the media $\mathrm{pH}$, electrostatic repulsion between $\mathrm{CF}$ and the films also increased, which accelerated the release of $\mathrm{CF}$ from the films. As for $\mathrm{MB}$, an increase in $\mathrm{pH}$ may have increased the interactions between $\mathrm{MB}$ and the films, which resulted in a decrease in the release rates. Since the expected results were not observed, the effect of swelling of the films seemed more significant than such interactions for MB. The results demonstrated that effect of media $\mathrm{pH}$ on the dye release properties appeared different depending on the molecular structures of the dyes.

\section{CONCLUSIONS}

Dye-loaded freestanding polysaccharide composite films were successfully obtained and the films were shown to exhibit media-responsive dye release abilities. The present results on the properties of these films demonstrate that they are promising implantable materials that can be used for wound dressing, cancer therapy, fomentation and in plasters that are put in contact with skin. Such films may also be useful as scaffolds in tissue engineering. Further systematic investigations on the effects of molecular sizes on the loading and release behaviors of films will take the current system to the next stage; for example, the design and preparation of the films could result in desirable spatiotemporal material release abilities. Enzymatic degradation of the polysaccharides can be used as a trigger for material release. Preparation of the films at lower temperatures is another interesting target in the preparation of films with protein release abilities.

\section{CONFLICT OF INTEREST}

The authors declare no conflict of interest.

\section{ACKNOWLEDGEMENTS}

This work was partially supported by JSPS KAKENHI Grant Numbers 22550155, 25410178, and by a grant from the Association for the Progress of New Chemistry (ASPRONC), Japan.

1 Stuart, M. A. C., Huck, W. T. S., Genzer, J., Muller, M., Ober, C., Stamm, M., Sukhorukov, G. B., Szleifer, I., Tsukruk, V. V., Urban, M., Winnik, F., Zauscher, S., Luzinov, I. \& Minko, S. Emerging applications of stimuli-responsive polymer materials. Nat. Mater. 9, 101-113 (2010).
2 Wolinsky, J. B., Colson, Y. L. \& Grinstaff, M. W. Local drug delivery strategies for cancer treatment: gels, nanoparticles, polymeric films, rods, and wafers. J. Controlled Release 159, 14-26 (2012).

3 Siepmann, F., Siepmann, J., Walther, M., MacRae, R. J. \& Bodmeier, R. Polymer blends for controlled release coatings. J. Controlled Release 125, 1-5 (2008).

4 Zilberman, M. \& Elsner, J. J. Antibiotic-eluting medical devices for various applications. J. Controlled Release 130, 202-215 (2008).

5 Rinaudo, M. Main properties and current applications of some polysaccharides as biomaterials. Polym. Int. 57, 397-430 (2008).

6 lijima, K. \& Hashizume, M. Application of polysaccharides as structural materials. Trends Glycosci. Glycotechnol. 27, 67-79 (2015).

7 Czaja, W. K., Young, D. J., Kawecki, M. \& Brown, R. M. Jr. The future prospects of microbial cellulose in biomedical applications. Biomacromolecules 8, 1-12 (2007).

8 Ravi Kumer, M. N. V., Muzzarelli, R. A. A., Muzzarelli, C., Sashiwa, H. \& Domb, A. J. Chitosan chemistry and pharmaceutical perspectives. Chem. Rev. 104, 6017-6084 (2004)

9 Sintov, A., Di-Capua, N. \& Rubinstein, A. Cross-linked chondroitin sulphate: characterization for drug delivery purposes. Biomaterials 16, 473-478 (1995).

10 Kubo, K. \& Kuroyanagi, Y. Development of cultured dermal substitute composed of a spongy matrix of hyaluronic acid and atelo-collagen combined with fibroblasts: cryopreservation. Artif. Organs 28, 182-188 (2004).

11 Vercruysse, K. P., Marecak, D. M., Marecek, J. F. \& Prestwich, G. D. Synthesis and in vitro degradation of new polyvalent hydrazide cross-linked hydrogels of hyaluronic acid. Bioconjugate Chem 8, 686-694 (1997).

12 Luo, Y., Kirker, K. R. \& Prestwich, G. D. Cross-linked hyaluronic acid hydrogel films: new biomaterials for drug delivery. J. Controlled Release 69, 169-184 (2000).

13 Hunt, J. A., Joshi, H. N., Stella, V. J. \& Topp, E. M. Diffusion and drug release in polymer films prepared from ester derivatives of hyaluronic acid. J. Controlled Release 12, 159-169 (1990).

14 Campoccia, D., Doherty, P., Radice, M., Brun, P., Abatangelo, G. \& Williams, D. F. Semisynthetic resorbable materials from hyaluronan esterification. Biomaterials 19 , 2101-2127 (1998).

15 Hashizume, M., Kobayashi, H. \& Ohashi, M. Preparation of Free-Standing Films of Natural Polysaccharides Using Hot Press Technique and Their Surface Functionalization with Biomimetic Apatite. Colloid Surf. B: Biointerfaces $\mathbf{8 8}$, 534-538 (2011).

16 Hashizume, M., Ohashi, M., Kobayashi, H., Tsuji, Y. \& Iijima, K. Preparation of Free-Standing Polysaccharide Composite Films: Improved Preparation and Physical Properties. Colloid Surf. A: Physicochem. Eng. Aspects 483, 18-24 (2015).

17 Iijima, K., Yuyama, K., Asaine, K., Irie, K. \& Hashizume, M. Preparation of Chondroitin Sulfate/Chitosan Composite Fibers by Spinning from Aqueous Solution Interfaces. Kobunshi Ronbunshu 71, 11-16 (2014).

18 Chen, W.-B., Wang, L.-F., Chen, J.-S. \& Fan, S.-Y. Characterization of Polyelectrolyte Complexes between Chondroitin Sulfate and Chitosan in the Solid State. J. Biomed. Mater. Res. 75A, 128-137 (2005).

19 Kikuchi, Y. \& Noda, A. Polyelectrolyte complexes of heparin and chitosan. J. Appl. Polym. Sci. 20, 2561-2563 (1976).

20 Yeh, M.-K., Cheng, K.-M., Hu, C.-S., Huang, Y.-C. \& Young, J.-J. Novel protein-loaded chondroitin sulfate-chitosan nanoparticles: preparation and characterization. Acta Biomater. 7, 3804-3812 (2011).

21 Bergmann, K. \& O'Konski, C. T. A spectroscopic study of methylene blue monomer, dimer, and complexes with montmorillonite. J. Phys. Chem. 67, 2169-2177 (1963).

22 Meng, X., Tian, F., Yang, J., Hem, C.-N., Xian, N. \& Li, F. Chitosan and alginate polyelectrolyte complex membranes and their properties for wound dressing application. J. Mater. Sci. Mater. Med 21, 1751-1759 (2010).

23 Domard, A. pH and c.d. measurements on a fully deacetylated chitosan: application to Cull-polymer interactions. Int. J. Biol. Macromol. 9, 98-104 (1987).

24 Rujiravanit, R., Kruaykitanon, S., Jamieson, A. M. \& Tokura, S. Preparation of crosslinked chitosan/silk fibroin blend films for drug delivery system. Macromol. Biosci. 3, 604-601 (2003).

25 Chen., J.-X., Wang, M., Tian, H.-H. \& Chen., J.-H. Hyaluronic acid and polyethyleneimine self-assembled polyion complexes as $\mathrm{pH}$-sensitive drug carrier for cancer therapy. Colloids Surf. B: Biointerfaces 8, 81-87 (2015). 